\title{
Breast Biopsy pathological technique and diagnostic application
}

\author{
Yanli LV \\ Department of xxx, People's Hospital of Zhengzhou, Zhengzhou 450000, Henan Province, \\ P.R.China \\ email: tuke126@126.com \\ Corresponding Author: Yanli LV
}

Keywords: Breast Disease, Biopsy, Pathological technique.

\begin{abstract}
The diagnosis of breast disease in Europe and other advanced western developed countries were mainly the biopsy technique, which included fine-needle aspiration biopsy for cytological diagnosis and core needle biopsy for histological diagnosis. This two methods could figure out the basic pathological lesions qualitative and histological classification of breast disease. Due to various reasons limitation, it had not been widely applied. The excision surgery and incisional biopsy was still existed, which brought more pain to patients, and increased the economic burden.
\end{abstract}

\section{Information and Method}

General Information

From January 2011 to December 2013, there were 302 cases of hospitalized patients had been performed breast biopsy in People's Hospital of Zhengzhou. The retrospective analysis had applied for the research. Preoperative X-ray mammography screening was employed under ultrasound machine for breast biopsy.

FNAB Pathology Technical Operation Method

Under aseptic operation, the disinfection was performed with the center of needle aspiration, with one hand fixing the lump and compacting skin, and another hand holding needle and piercing lump against the chest wall in a certain angle. The best depth of needle pierce should be the two-thirds diameter of lump. Negative pressure was made by suction, suction repeatedly several times, the needle suction angle could also be changed. When the lump material was inhaled into the needle. When bolt was in normal pressure or slightly negative pressure, the needle was withdrew. Sterile cotton ball was pressed on needle puncture point, then fixed with tape. The sucked-out content was injected on glass slide, smear on 4 to 5 slides, then dried for few minutes, then went for HE staining. The observation and diagnosis was performed under microscopic instrument.

\section{CNB Technical Operation Method}

In the ultrasound with high frequency $7.5 \sim 18 \mathrm{mHz}$ guidance, $14 \mathrm{G}$ needle with diameter of $1.63 \mathrm{~mm}$ or $16 \mathrm{G}$ needle was chosen, and with range of $15 \mathrm{~mm}$ or $21 \mathrm{~mm}$. Two-dimensional ultrasound was chosen first to detect the tumor size, morphology, boundary, envelope, echo changes and lymph nodes. Antiseptic wipes was flatly spread. Sterile probe scanning was also performed. Needle was injected under the local anesthesia, biopsy gun was released quickly. After the withdraw, the lump tissue was fixed in formalin liquid. The needle puncture area was pressed 5 to 10 minutes for hemostasis, the pneumothorax presence or not was monitored. Different directions or parts was taken 2 to 5 pieces. Biopsy was the rapid paraffin processing, and drawn the report in the same day.

\section{Statistical Method}

SPSS software was applied for statistical analysis, percentage comparison was the chi-square test, $\mathrm{P}<0.05$ was for the statistically difference significance. 


\section{Result}

\section{Data of This Group}

In the 302 cases of breast biopsy patients, the oldest was 83 years old, the youngest was 18 years old, the average is 44.8 years old; 246 cases of patients were married, accounting for $81.5 \%$, unmarried patients was 56 cases, accounting for 18.5\%; there were 164 cases of patients with occupation of government institutions, accounting for 54.3\%, 79 cases of patients with profession of business operators or staff, accounting for $26.2 \%$, and 59 cases of house workers, account for $19.7 \%$; 76 cases with medium education or below, accounting for $25.2 \%, 226$ cases of patients with education of undergraduate course or above, accounting for $74.8 \%$.

\section{Breast Disease Detection}

The fine-needle aspiration biopsy, also named FNAB, and core needle biopsy, CNA were employed to detect the breast cancer disease benign lesion, atypical hyperplasia, malignant lesion and uncertain lesion, details was seen in table 1.

Table 1. HE dyeing of benign and malignant results for 302 cases of breast disease biopsy specimens (n, \%)

\begin{tabular}{|l|l|l|l|l|l|}
\hline $\begin{array}{l}\text { Detection } \\
\text { method }\end{array}$ & $\mathrm{n}$ & Benign lesion & Atypical hyperplasia & Malignant lesion & uncertain lesion \\
\hline FNAB & 58 & $19(32.8 \%)$ & $3(5.2 \%)$ & $29(50.0 \%)$ & $7(12.1 \%)$ \\
\hline CNB & 244 & $82(33.6 \%)$ & $22(9.0 \%)$ & $135(55.3 \%)$ & $5(2.0 \%)$ \\
\hline & 302 & $101(33.4 \%)$ & $25(8.3 \%)$ & $164(54.4 \%)$ & $12(3.9 \%)$ \\
\hline
\end{tabular}

\section{Breast Disease Histological Classification}

Through HE staining diagnosis, there were malignant lesion in 164 cases, including invading ductal carcinoma 116 cases, accounting for 70.7\%, ductal carcinoma and severe atypical hyperplasia in 28 cases for $17.1 \%$, mucinous adenoarcinoma in 9 cases for $5.5 \%$, metastatic invading carcinoma in 8 cases for $4.9 \%$, medullary carcinoma in 2 cases for $1.2 \%$, cystosarcoma phylloides in 1 cases for $0.6 \%$. There were benign lesion in $101 \mathrm{c}$ ases, including fibroadenoma in 43 cases for $42.6 \%$, breast adenosis in 31 cases for $30.7 \%$, intraductal papilloma in 12 cases for 11.9\%. Breast plasma cells mastitis in 6 cases for 5.9\%, granulomatous inflammation in 5 cases for $5.0 \%$, tuberculous abscess in 4 cases for $4.0 \%$.

\section{Discussion}

At present, breast disease pathological diagnosis were mainly FNAB, NAB, Mammtome and breast lump removal or incision operation [1]. From the table 1, in the 302 cases of specimens, the FNAB and NAB diagnosed benign lesion percentage were respectively $32.8 \%$ and $33.6 \%$. The malignant were respectively $50.5 \%$ and $55.3 \%$. These two diagnosis methods had no significant difference, $\mathrm{P}>0.05$. While, CNB unconfirmed cases was $2.0 \%$, FNAB unconfirmed ratio was $12.1 \%$. The ratio of two method comparative differences were statistical significant, $\mathrm{P}<0.01$, which indicated diagnostic accurate rate of CNB was obviously higher than that of FNAB.

In recent 10 years, CNB technology was widely applied internationally for breast disease diagnosis. Form the table 1, most disease could be diagnosed for benign and malignant disease and histological classification, excluding few cases of unconfirmed disease. In the hospitalized patients during the 2 years, the first three malignant tumor of female breast disease were invading ductal carcinoma for $70.7 \%$, intraductal carcinoma for $17.1 \%$ and mucinous adenoarcinoma for $5.5 \%$; the first three benign disease were fibroadenoma for $42.6 \%$, mammary gland disease for $30.7 \%$ and intraductal papiloma for $11.9 \%$. The biggest characteristic of CNB were that the serial section was suitable for immuohistochemical detection, sex hormone receptor detection, Her2 detection, DNA quantitative measurement, in order to meet the needs of the clinical diagnosis and treatment of mammary gland disease, such as the secretion of breast cancer treatment, neoadjuvant chemotherapy scheme selection and operation method judgment. Nowadays, biopsy of breast carcinoma metastases were also increasingly popular with the clinical doctors, because the studies have proved that the changes of metastases receptor detection ER, PR and CerbB-2 condition could 
respectively alter the treatment method for $20 \%$ and $12 \%$ of patients treatment $[2,3]$.

CNB method could be gained 2 to 4 hours of fast paraffin wax production. Biopsy specimen embedding should be paid attention to the tissue being fixed parallel, and set in the center area and in the same horizontal plane, and clipped tissue should be gently, avoiding the tissue fracture; Peeling the slice should be gently, and slowly uniform serial section, so fast cutting could occur cavitation [4]; it need to ensure the maximum tissue section, the glass slide can be 6 to 8 pieces, and avoid the missing inspection.

CNB could gain more tissues than FNAB. It was still dispute that whether the disease tissue, especially the malignant tissue still remained in the needle hole after repeated puncture. However, there some studies proved that there was no statistical significance for local tumor recurrence rate of CNB and surgical excision biopsy. Breast conserving surgery was performed before radiotherapy, and CND did not affect survival rate [5]. The appropriate puncture point should be chosen, as it would be better remove the puncture point area and needle hole. FNAB was the pathology technique applied on breast disease diagnosis and screening since 1950s, with the advantage of simple and safe operation and low expenditure [6]. In the case of clinical and radiological technique indicated disease, FNAB could be applied on preliminary clinical diagnosis. FNAB could basically distinguish benign and malignant disease. European and American countries have adopted similar 5 degree of magnitude [7]. While, China was still lack of FNAB result interpretation standards. There still existed matters, such as false negatives, less material, unable to distinguish situ carcinoma and invasion carcinoma. CNB has some issues to settle too. The tumor with indicating of imaging still could have negative results, the reason may due to the extra small size of tumor, biopsy needle cannot read the designated position, or disease tissue containing hard calcified components, needle cannot pierce in, larger size lump with local partial malignant lesion could cause misdiagnosis [8].

In conclusion, under the guidance of ultrasound, CNB was more worthy of promotion than FNAB, Mammotome and lump removal method for breast disease, due to the little injury, low expenditure, high sensitivity and accuracy, more reliable diagnosis. it provided theoretical basis for prevention and screen of breast disease.

\section{References}

[1] Gonzalea E, Grafton WD, Mirris DM, et al. Diagnosing breast cancer using frozen section form Tue-cut needle biopsies[J]. Ann Surg,1998,202(6):696.

[2] Simmons C, Miller N, Geddie M, et al. Does confirmation tumor biopsy alter the management of breast cancer patients with distant metastases[J]. Ann Oncol,2009,20(9):1499.

[3] Amir E, Freedman O, Simmons C, et al. Biopsy confirmation of metastatic disease in breast cancer: results from a large prospective study[J]. Cancer Res, 2009,69(24):2023.

[4] Ma HH, Zhou XJ. Biopsy common problems and countermeasures[J]. Chinese Journal of Clinical and Experimental Pathology, 2009,25(4):211.

[5] Takaki K, Sasano H, Ishida T, et al. Comparison of core needle biopsy(CNB) and surgical specimens of breast cancer patients[J]. Cancer Sci, 2010,101(9):2074.

[6] Graces CA, Cance WG. Neoadjuvant chemotherapy of breast cancer[J]. Ann Surg, 2004,70(7): 565.

[7] Dash N, Chafin S, Johnson RR, et al. Usefulness of tissue marker clips in patients undergoing Neoadjuvant chemotherapy for breast cancer[J]. AJR Am Roentgenol,1999,173(4):911.

[8] Youk J, kim EK, Kim MJ, et al. Analysis of false-negative results saffer US-guided 14G core needle breast biopsy[J]. Ur Radiol,2010,20(4):782. 